\title{
Origin of two-hole triplet splitting in circular quantum dots
}

\author{
Juan I. Climente \\ Departament de Química Fúsica i Analítica, Universitat Jaume I, E-12080, Castelló, \\ Spain
}

\begin{abstract}
Recent photoluminescence spectra of positively charged excitons in InAs/GaAs quantum dots have revealed the existence of large splittings between the pshell triplet sublevels of holes. We provide an intuitive explanation for their origin in terms of heavy hole-light hole coupling using Luttinger spinors. These splittings are present even in symmetric quantum dots at zero field and their magnitude can be tuned by the geometry of the dot. We show that the spin purity of the triplet drastically decreases with the aspect ratio, but that of the singlet ground state remains high.
\end{abstract}

Keywords: semiconductors, nanostructures, spin-orbit effects, electronic band structure, electron-electron interactions

Email address: climente@uji.es (Juan I. Climente)

$U R L:$ http://quimicaquantica.uji.es/ (Juan I. Climente) 


\section{Introduction}

Negative trions with one electron in the s-shell and another in the p-shell, often referred to as p-shell trions or hot trions, have been proposed for optical electron spin polarization and spin memory devices based on quantum dots (QDs).[1, 2, 3] These applications rely on the fine structure of the triplet sublevels, which are split by tens of $\mu \mathrm{eV}$ even at zero magnetic field due to the electron-hole exchange interaction, each set of sublevels having a distinct response to circularly polarized light. [1, 4]

The same fine structure was expected for positive trions, [5] but recent optical measurements reveal the presence of an additional number of resonances and large $(\sim \mathrm{meV})$ splittings, which cannot be accounted for by the weak electron-hole exchange interaction alone.[6, 7, 8] Warming and co-workers showed that these splittings could be correctly reproduced if one considers anisotropic hole-hole exchange interaction and Coulomb correlations. [6] The underlying idea is that piezoelectric fields lower the otherwise circular symmetry of self-assembled InAs/GaAs QDs. Because the valence band spin-orbit interaction is significant, the hole-hole spin exchange interaction is sensitive to the spatial asymmetry and hence degeneracies are lifted. Soon after it was realised that single-hole p-shell energy levels in InAs/GaAs QDs also present few-meV splittings. $[9,10]$ These splittings could be easily understood if the lateral confinement was anisotropic, e.g. due to an elongation of the QD base or to piezoelectric fields.[11, 12] However, the magnitude observed is too large for the expected degree of anisotropy. Using an 8-band k.p Hamiltonian with realistic simulation of the inhomogeneous QD composition, strain and piezoelectric fields, it was then shown that heavy hole-light hole (HH-LH) coupling was the main factor contributing to such a splitting. [9] Furthermore, it was found that HH-LH coupling increased with the vertical aspect ratio, which translated into enhanced p-shell splittings. These results were however of numerical nature, and a more profound understanding is of interest to gain control on the electronic structure of p-shell holes and positive trions in QDs. This is the goal of the present work.

We study holes and trions in cylindrical InGaAs QDs. Circular dots are not only of fundamental interest - realistic self-assembled QDs often present but small deviations from this ideal limit-, but also of practical importance, as highly symmetric QDs are desirable for spintronic applications to minimize spin admixture through spin-orbit interaction. [13, 14, 15] Holes are described as Luttinger spinors resulting from a 4-band $\mathrm{k} \cdot \mathrm{p}$ Hamiltonian. We find that 
HH-LH coupling gives rise to large splittings of the p-shell even if the QDs have perfect circular symmetry, and show that this follows from the unequal centrifugal energy of the minor components in $p_{+}$and $p_{-}$states. The dependence of the splitting on the vertical aspect ratio is then easily understood from the different effective masses of HHs and LHs. We next show this pshell splitting gives rise to a non-negligible splitting of the two-hole triplet, which is clearly reflected in the optical spectrum of hot trions, as noted in related experiments. $[6,7,8]$ Because HH-LH coupling is so influential in these systems, we also investigate whether it has a significant impact on the spin purity of the hot trion, which could affect the performance of positive trions in practical applications. [7] We find that the spin purity of the excited triplet rapidly decreases with increasing aspect ratio, but that of the singlet remains high. This has implications for optical spin control which we discuss. Last, we show that the hole triplet splitting is also responsible for the appearance of multiple resonances in the emission of doubly charged excitons.[23]

\section{Theory}

The general expression of our Hamiltonian can be written in the second quantization as:

$$
\begin{aligned}
\hat{H}=\sum_{i} E_{i}^{e} e_{i}^{+} & e_{i}+\sum_{p} E_{p}^{h} h_{p}^{+} h_{p}+\frac{1}{2} \sum_{i j k l}\langle i j|V| k l\rangle e_{i}^{+} e_{j}^{+} e_{k} e_{l} \\
& +\frac{1}{2} \sum_{p q r s}\langle p q|V| r s\rangle h_{p}^{+} h_{q}^{+} h_{r} h_{s}+\sum_{i j p q}\langle i p|V| q j\rangle e_{i}^{+} h_{p}^{+} h_{q} e_{j},
\end{aligned}
$$

where $E_{i}^{e}\left(E_{p}^{h}\right)$ is the electron (hole) energy in the single-particle state $|i\rangle$ $(|p\rangle), e_{i}^{+} / e_{i}\left(h_{p}^{+} / h_{p}\right)$ is the electron (hole) creation/annihilation operator, i.e., $\left(e_{i}^{+} e_{j}^{+} \ldots\right)\left(h_{p}^{+} h_{q}^{+} \ldots\right)\left|0_{h}\right\rangle\left|0_{e}\right\rangle=|p q \ldots\rangle|i j \ldots\rangle$, and $\langle i j|V| k l\rangle,\langle p q|V| r s\rangle$ and $\langle i p|V| q j\rangle$ are the electron-electron, hole-hole and electron-hole Coulomb matrix elements respectively.

The single-particle electron and hole states are calculated as in Ref. [16]. Electrons are modeled using a single-band effective mass Hamiltonian. For QDs with $C_{\infty v}$ symmetry and negligible spin-orbit interaction, the states are of the form:

$$
\left|m_{z}, n, \sigma\right\rangle=f_{m_{z}, n}(\rho, z)|\sigma\rangle
$$


where $m_{z}$ is the azimuthal angular momentum of the envelope function, $n$ is the main quantum number and $\sigma=\uparrow, \downarrow$ the spin projection and $f_{m_{z}, n}(\rho, z)$ the envelope function.

For holes, spin-orbit interaction is significant and the spin is no longer a good quantum number. Instead, the Bloch total angular momentum $J_{z}$ is used.[17] For HHs, $J_{z}= \pm 3 / 2$ and the periodic Bloch functions are given by:

$$
\begin{aligned}
& \left|J_{z}=+3 / 2\right\rangle=\frac{-1}{\sqrt{2}}\left|P_{+}\right\rangle|\uparrow\rangle, \\
& \left|J_{z}=-3 / 2\right\rangle=\frac{1}{\sqrt{2}}\left|P_{-}\right\rangle|\downarrow\rangle .
\end{aligned}
$$

where $P_{ \pm}=|X\rangle \pm i|Y\rangle$. For LHs, $J_{z}= \pm 1 / 2$ and the Bloch functions are:

$$
\begin{aligned}
\left|J_{z}=+1 / 2\right\rangle & =\sqrt{\frac{2}{3}}\left|P_{z}\right\rangle|\uparrow\rangle-\frac{1}{\sqrt{6}}\left|P_{+}\right\rangle|\downarrow\rangle, \\
\left|J_{z}=-1 / 2\right\rangle & =\sqrt{\frac{2}{3}}\left|P_{z}\right\rangle|\downarrow\rangle+\frac{1}{\sqrt{6}}\left|P_{-}\right\rangle|\uparrow\rangle .
\end{aligned}
$$

We use a four-band k.p Luttinger-Kohn Hamiltonian coupling $\mathrm{HH}$ and LH subbands, so that hole states are described by four-component objects (Luttinger spinors). Each component is given by a Bloch function $\left|J_{z}\right\rangle$ and an envelope function $f_{m_{z}, n}^{J_{z}}(\mathbf{r})$. In the presence of axial symmetry, the envelope function has a well-defined angular momentum $m_{z}$, and the spinor can be classified by its total angular momentum $F_{z}=m_{z}+J_{z}$. A general hole spinor reads:

$$
\left|F_{z}, n\right\rangle=\left(\begin{array}{l}
f_{F_{z}-3 / 2, n}^{3 / 2}(\rho, z)\left|J_{z}=+\frac{3}{2}\right\rangle \\
f_{F_{z}-1 / 2, n}^{1 / 2}(\rho, z)\left|J_{z}=+\frac{1}{2}\right\rangle \\
f_{F_{z}+1 / 2, n}^{-1 / 2}(\rho, z)\left|J_{z}=-\frac{1}{2}\right\rangle \\
f_{F_{z}+3 / 2, n}^{-3 / 2}(\rho, z)\left|J_{z}=-\frac{3}{2}\right\rangle
\end{array}\right)
$$

where $n$ is the main quantum number.

Coulomb matrix elements are calculated as 5-dimensional integrals which we evaluate using adaptative Monte-Carlo routines.[18, 19] Many-body states are then computed with a configuration interaction (CI) routine.[20]

We consider a cylindrical QD with radius $R=15 \mathrm{~nm}$ and variable height $H$. InGaAs material parameters are taken for the numerical calculations: the electron effective mass is $m^{*}=0.05 m_{0}$, where $m_{0}$ is the free electron 
mass; $\gamma_{1}=11.01, \gamma_{2}=4.18$ and $\gamma_{3}=4.84$ are the Luttinger parameters and $\varepsilon=12.9$ the dielectric constant.[21] The confining potential outside the QD is set by the InGaAs/GaAs band-offset. We take $V=0.5 \mathrm{eV}$ for electrons and $V=0.3 \mathrm{eV}$ for holes.

\section{Results}

\subsection{Single carrier}

We start by comparing the shell structure of single electrons and holes in Figure 1. For electrons, Fig. 1a, the s-shell is formed by $\left|m_{z}, \sigma\right\rangle=|0, \uparrow\rangle$ and $|0, \downarrow\rangle$. The p-shell is formed by two $\mathrm{p}_{-}$states $(|-1, \uparrow\rangle,|-1, \downarrow\rangle)$ and two $\mathrm{p}_{+}$states $(|+1, \uparrow\rangle,|+1, \downarrow\rangle)$. This scheme reflects the usual degeneracies resulting from circular and time-reversal symmetries.

For holes, if we take into account HH-LH coupling, spin-orbit interaction lifts degeneracies and only Kramers-degenerate pairs are observed. The shell structure now resembles that of Fig. $1 \mathrm{~b}$. The s-shell is given by $F_{z}= \pm 3 / 2$ states, whose spinors read:

$$
\left|\frac{3}{2}, 1\right\rangle=\left(\begin{array}{c|c}
\mathbf{f}_{\mathbf{0 , 1}}^{\mathbf{3} / 2}\left|+\frac{\mathbf{3}}{\mathbf{2}}\right\rangle \\
f_{1,1}^{1 / 2}\left|+\frac{1}{2}\right\rangle \\
f_{2,1}^{-1 / 2}\left|-\frac{1}{2}\right\rangle \\
f_{3,1}^{-3 / 2}\left|-\frac{3}{2}\right\rangle
\end{array}\right) \quad\left|\frac{-3}{2}, 1\right\rangle=\left(\begin{array}{c}
f_{-3,1}^{3 / 2}\left|+\frac{3}{2}\right\rangle \\
f_{-2,1}^{1 / 2}\left|+\frac{1}{2}\right\rangle \\
f_{-1,1}^{-1 / 2}\left|-\frac{1}{2}\right\rangle \\
\mathbf{f}_{\mathbf{0}, \mathbf{1}}^{-3 / 2}\left|-\frac{\mathbf{3}}{\mathbf{2}}\right\rangle
\end{array}\right)
$$

The first excited states are those with $F_{z}= \pm 1 / 2$, whose spinors are:

$$
\left|\frac{1}{2}, 1\right\rangle=\left(\begin{array}{c}
\mathbf{f}_{-\mathbf{1 , 1}}^{\mathbf{3} / \mathbf{2}}\left|+\frac{\mathbf{3}}{\mathbf{2}}\right\rangle \\
f_{0,1}^{1 / 2}\left|+\frac{1}{2}\right\rangle \\
f_{1,1}^{-1 / 2}\left|-\frac{1}{2}\right\rangle \\
f_{2,1}^{-3 / 2}\left|-\frac{3}{2}\right\rangle
\end{array}\right) \quad\left|\frac{-1}{2}, 1\right\rangle=\left(\begin{array}{c}
f_{-2,1}^{3 / 2}\left|+\frac{3}{2}\right\rangle \\
f_{-1,1}^{1 / 2}\left|+\frac{1}{2}\right\rangle \\
f_{0,1}^{-1 / 2}\left|-\frac{1}{2}\right\rangle \\
\mathbf{f}_{\mathbf{1 , 1}}^{-\mathbf{3}}\left|-\frac{\mathbf{3}}{\mathbf{2}}\right\rangle
\end{array}\right)
$$

Last, the second excited states are those with $F_{z}= \pm 5 / 2$, whose spinors are:

$$
\left|\frac{5}{2}, 1\right\rangle=\left(\begin{array}{c|c}
\mathbf{f}_{\mathbf{1}, \mathbf{2}}^{\mathbf{3}}\left|+\frac{\mathbf{3}}{\mathbf{2}}\right\rangle \\
f_{2,1}^{1 / 2}\left|+\frac{1}{2}\right\rangle \\
f_{3,1}^{-1 / 2}\left|-\frac{1}{2}\right\rangle \\
f_{4,1}^{-3 / 2}\left|-\frac{3}{2}\right\rangle
\end{array}\right) \quad\left|\frac{-5}{2}, 1\right\rangle=\left(\begin{array}{c}
f_{-4,1}^{3 / 2}\left|+\frac{3}{2}\right\rangle \\
f_{-3,1}^{1 / 2}\left|+\frac{1}{2}\right\rangle \\
f_{-2,1}^{-1 / 2}\left|-\frac{1}{2}\right\rangle \\
\mathbf{f}_{-\mathbf{1 , 1}}^{-\mathbf{3}}\left|-\frac{\mathbf{3}}{\mathbf{2}}\right\rangle
\end{array}\right)
$$


Boldface characters in the expressions above denote the dominant component of each spinor (i.e. the $\mathrm{HH}$ with lowest angular momentum). If only this component was present, as in usual single-band $\mathrm{HH}$ models, the shell structure would be identical to that of electrons (note that $\left|J_{z}= \pm 3 / 2\right\rangle$ functions contain pure spin projections, Eqs. 3,4). For instance, $F_{z}=1 / 2$ and $F_{z}=-5 / 2$ states would be equivalent to $m_{z}=-1$ states with $\sigma=\uparrow, \downarrow$, respectively. Nonetheless, the minor components of the spinors are different for each $\left|F_{z}\right|$. In particular, the minor components of $F_{z}=-5 / 2$ have larger $m_{z}$ than those of $F_{z}=1 / 2$. This results in larger centrifugal energy, which is roughly given by $\left\langle\left(m_{z} / \rho\right)^{2}\right\rangle$ ( $\rho$ is the radial coordinate). The lateral confinement of QDs leads to small $\langle\rho\rangle$ values, thus pushing $|-5 / 2,1\rangle$ higher in energy. We stress this occurs in spite of the perfect circular symmetry. In what follows, we refer to this zero-field p-shell splitting as $\Delta_{0}$.

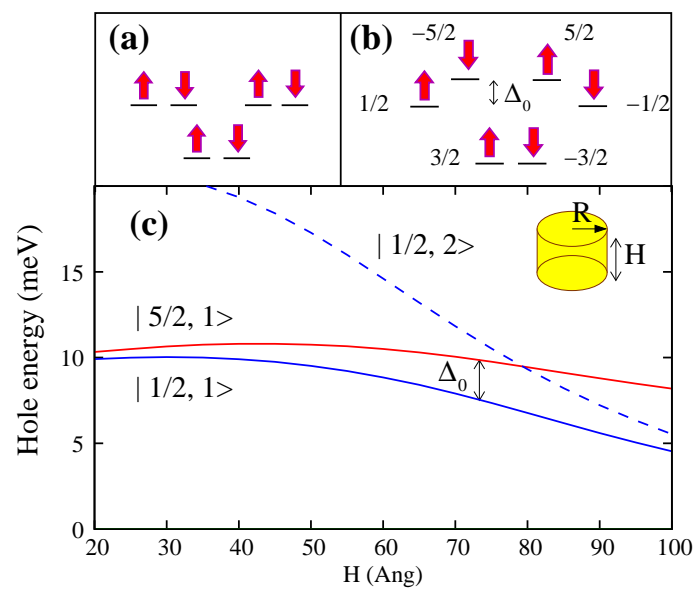

Figure 1: (a) Low-energy shell structure of a single electron. (b) Same of a single hole. $F_{z}$ quantum numbers are given. (c) Low energy holes states $\left|F_{z}, n\right\rangle$ as a function of the QD height. All energies are referred to that of $|3 / 2,1\rangle$.

To estimate the magnitude of $\Delta_{0}$, in Fig. 1c we plot the energy of the $|1 / 2,1\rangle$ and $|5 / 2,1\rangle$ (solid lines) as a function of the QD height. At $H=20$ $\AA, \Delta_{0}=0.4 \mathrm{meV}$ and it increases with the aspect ratio, reaching $\Delta_{0}=3.7$ meV at $H=100 \AA$. These values of the p-shell splitting are of the same order as, but smaller than, the $\sim 7 \mathrm{meV}$ reported in Ref. [9]. This is because the weight of the LH components in the idealized QDs we study (less than $6 \%)$ is lower than that estimated for the experimental samples $(\sim 10 \%)$. [9] 
At any rate, the trend observed in Fig. 1c is consistent with that of more sophisticated models, namelly the larger the aspect ratio the larger $\Delta_{0} .[9] \mathrm{A}$ simple explanation follows from our interpretation of the origin of $\Delta_{0}$. The effective mass of HHs along the [001] direction (growth direction) of zincblende crystals is $m_{h h}=1 /\left(\gamma_{1}-2 \gamma_{2}\right)$, which is heavier than that of LHs, $m_{l h}=1 /\left(\gamma_{1}+2 \gamma_{2}\right)$.[17] Thus, a strong vertical confinement implies large kinetic energies for LHs, which then couple more weakly to HH. The lowenergy hole spinors have then largely dominant $\mathrm{HH}$ character. Conversely, with decreasing vertical confinement strength, the LH character of the spinor increases, which enhances $\Delta_{0}$.

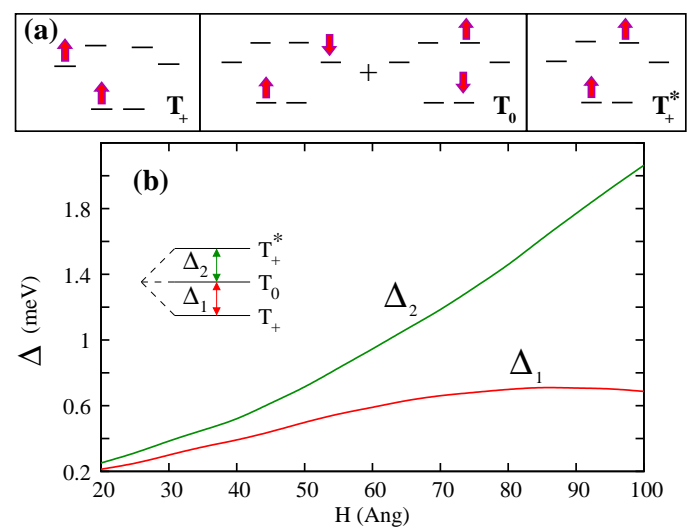

Figure 2: (a) Electronic configuration of three non-degenerate triplet sublevels. (b) Energy splitting between such sublevels as a function of the QD height.

\subsection{Two holes}

The p-shell splitting will certainly influence the hot trion energy structure. To understand how this occurs we first study the two-hole triplet. In a singleband picture, one hole would occupy a s-orbital and another a $\mathrm{p}_{+}$or $\mathrm{p}_{-}$ orbital. The spin degeneracy would allow for the three triplet projections, $\mathrm{T}_{+}, \mathrm{T}_{0}$ and $\mathrm{T}_{-}$. All in all, this yields six degenerate triplet sublevels at zero field. The presence of $\Delta_{0}$ however splits the triplets into three two-folddegenerate sublevels. Fig. 2a illustrates the dominant electronic configuration of three splitted triplet states. The lowest energy sublevels, $T_{ \pm}$, have total (two-body) angular momentum $F_{z}^{t}= \pm 2$, the $T_{0}$ sublevels $F_{z}^{t}= \pm 1$ and the topmost sublevels, $T_{+}^{*}, F_{z}^{t}=4$. Fig. $2 \mathrm{~b}$ shows the calculated magnitude 
of the splitting between triplet sublevels $\left(\Delta_{1}, \Delta_{2}\right)$ as a function of the QD height. One can see that the splittings tend to increase with the aspect ratio. Still, the magnitude is systematically smaller than that expected from a single-particle picture, where $\Delta_{1}=\Delta_{2}=\Delta_{0} / 2$. The deviation arises from Coulomb correlations, which affect $T_{ \pm}, T_{0}$ and $T_{ \pm}^{*}$ with different intensity.

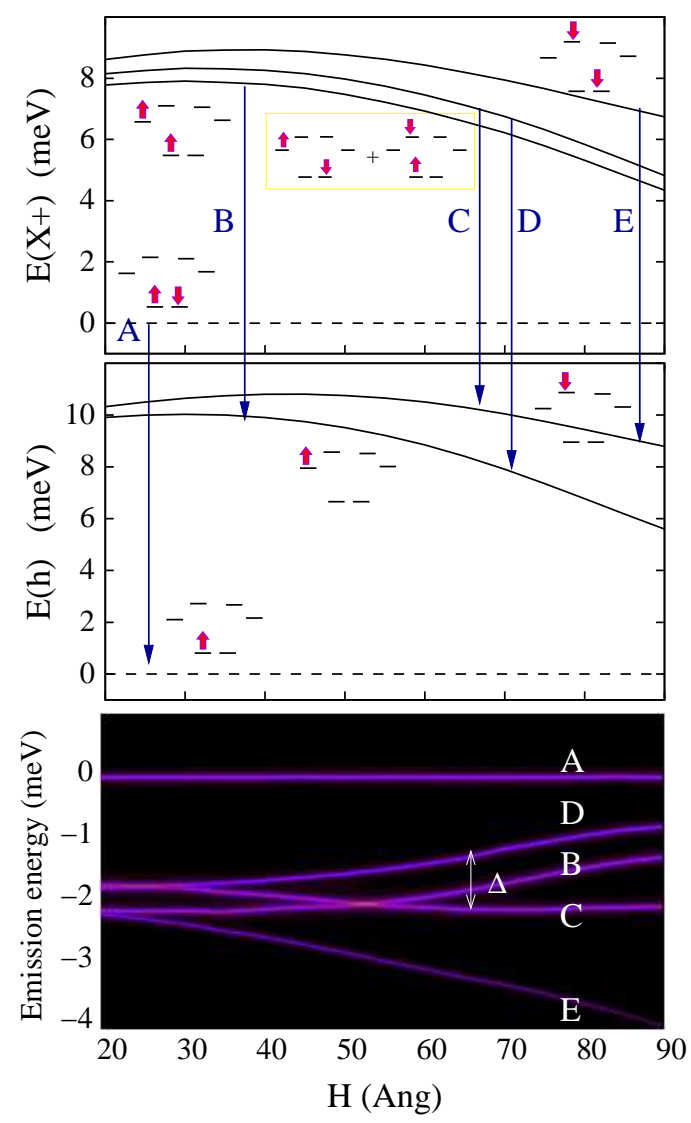

Figure 3: (a) Hot trion energy levels, (b) single-hole energy levels and (c) trion emission spectrum as a function of the QD height. All energies are referred to the lowest energy states (dashed lines). The schematics represent the dominant hole configurations.

\subsection{Hot trion}

We are now in a condition to investigate the hot trion properties. The emission spectrum is studied in Fig. 3. The initial positive trion states are 
shown in Fig. 3a, with schematics illustrating the dominant two-hole configuration. One can recognize the three split triplet sublevels well above the singlet ground state (dashed line). After the electron-hole recombination, the final state is given by a single hole, whose energy levels are shown in Fig. 3b. The emission spectrum is then calculated within the dipole approximation, as described in Ref. [22]. The resulting spectrum is plotted in Fig. 3c. The fundamental transition -between the trion and single-hole ground states- is labeled as A. The trion formed by a s-shell electron and the $T_{ \pm}$hole triplet gives rise to transition $\mathrm{B}$. That involving $T_{0}$ gives rise to transitions $\mathrm{C}$ and $\mathrm{D}$, and that involving $T_{ \pm}^{*}$ gives rise to transition $\mathrm{E}$. It is worth noticing that the resonances corresponding to transitions B-E are non-degenerate, contrary to the spectrum expected from single-band models, and is consistent with optical measurements of positive trions, which reveal various resonances under the fundamental transition. $[6,7,8]$ Note also that $\mathrm{C}$ and $\mathrm{D}$ resonances differ only in the final hole state, so their splitting provides a direct measurement of the p-shell splitting, $\Delta_{0}$. Yet, this resonance may be difficult to observe experimentally because $T_{0}$ tends to thermalize quickly to the singlet ground state.[1,3] The use of electrical charging control may facilitate this task. [4]

Electron-hole exchange, which has been disregarded here for simplicity, will further split trion states with hole configurations $\mathrm{T}_{ \pm}$, and $\mathrm{T}_{ \pm}^{*}$ into dark and bright states.[5] This will induce small $(\mu \mathrm{eV})$ shifts in the energy of resonances $\mathrm{B}$ and $\mathrm{E}$ in Fig. 3. Anisotropic electron-hole exchange involving p-shell holes is negligible.[23]

Negatively charged hot trions have been proposed for optically controlled spin devices. $[1,2,3]$ These applications are supported by the high spin purity of electrons in QDs, which gives rise to strict spin selection rules and long spin lifetimes. [24] The suitability of positively charged trions is not clear a priori, as the valence band spin-orbit interaction can lead to significant hole spin mixing. [14, 25, 26] To address this question we calculate the expectation value of the hole spin in single-hole and two-hole states. The results are shown in Fig. 4a for different values of the QD height. Electronic configurations are denoted by $\left(\begin{array}{c}e_{S}, e_{P} \\ h_{S}, h_{P}\end{array}\right)$, where $e_{S}\left(e_{P}\right)$ stands for an electron in the s- $(\mathrm{p}-)$ shell and $h_{S}\left(h_{P}\right)$ for a hole in the s-(p-)-shell (actually, $\left|F_{z}\right|=3 / 2$ and $\left.\left|F_{z}\right|=1 / 2\right)$. $\uparrow$ and $\downarrow$ are the positive and negative electron spin $(\sigma)$ projections, while $\Uparrow$ and $\Downarrow$ are the positive and negative hole pseudospin $\left(F_{z}\right)$ projections. One can see that single holes in the s-shell (dashed lines) have expectation values near $\left\langle S_{z}\right\rangle \pm 1 / 2$ for all the range of geometries under study. This indicates that the Luttinger spinor is essentially a $\mathrm{HH}$, with well defined spin. Likewise, 

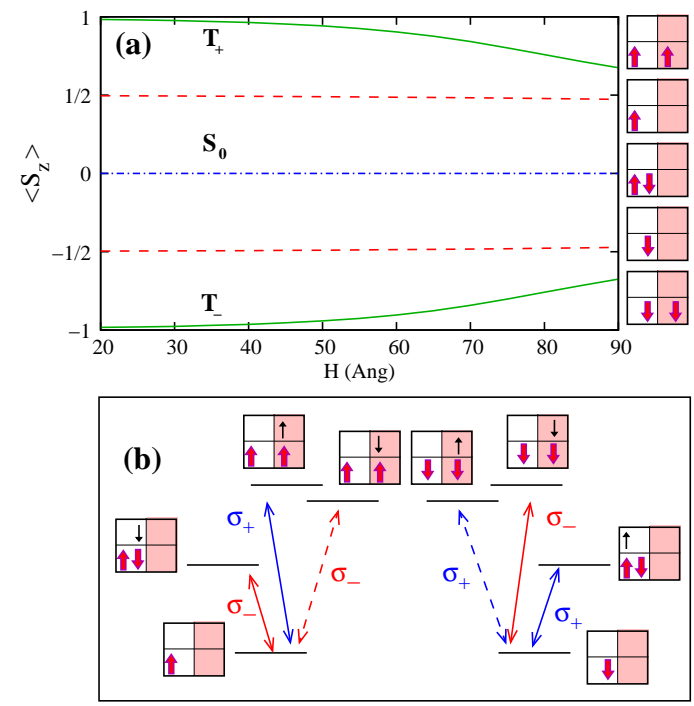

Figure 4: (a) Hole spin expectation value for different single-hole and two-hole states. The schematics on the left show the dominant electronic configuration. (b) Sketch of optical transitions from single-hole to positive trion $T_{ \pm}$states mediated by left $\left(\sigma_{-}\right)$or right $\left(\sigma_{+}\right)$ circularly polarized light. Unshaded (shaded) boxes represent s (p) states. In (b), dashed lines correspond to spin-forbidden transitions, which are enabled by spin mixing in high aspect ratio QDs.

the two-hole singlet $\left(\begin{array}{c}0,0 \\ \Uparrow \Downarrow, 0\end{array}\right)$ has $\left\langle S_{z}\right\rangle=0$, also denoting high spin purity. $\mathrm{T}_{ \pm}$ triplet states, $\left(\begin{array}{l}0,0 \\ \Uparrow, \Uparrow\end{array}\right)$ and $\left(\begin{array}{l}0,0 \\ \Downarrow, \Downarrow\end{array}\right)$, behave however differently (see solid lines). For small QD height, $\left\langle S_{z}\right\rangle= \pm 1$, but large deviations are observed upon increasing the height. This is because the LH components of the spinor gain weight, and they have mixed spin up and spin down projections, see Eqs. 5,6.

The results in Fig. 4a imply that typical self-assembled InGaAs QDs -where the aspect ratio is small- are suitable for spin-based operations relying on the positive trion, just like in negative trions. In QDs with large aspect ratio, however, the p-shell triplet suffers from severe spin mixing, which translates into weak spin selection rules and coupling to states which would otherwise require a spin flip mechanism. As sketched in Fig. 4b, one can still perform spin selective s-shell excitations from single-hole states to the trion singlet state using circularly polarized light, as done in Ref.[7]. This can be exploited e.g. for non-destructive spin readout measurements via cycling transitions.[15] By contrast, p-shell excitations to the hot trion are no 
longer as spin selective as in negative trions.[2] The large spin mixing renders optically active otherwise dark trion states, e.g. $\left(\begin{array}{l}0,0 \\ \Uparrow, 0\end{array}\right) \rightarrow\left(\begin{array}{l}0, \downarrow \\ \Uparrow, \Uparrow\end{array}\right)$ (dashed lines in Fig. 4b), and mixing between dark and bright subspaces leads to fast spin relaxation.[26]

\subsection{Doubly charged exciton}

The splitting of the hole triplet discussed in Section 3.2 is not only reflected in the emission spectrum of hot trions, but also in the that of doubly charged excitons. Ediger et al. compared the photoluminescence spectra of $\mathrm{X}^{2-}(3 \mathrm{e}+1 \mathrm{~h})$ and $\mathrm{X}^{2+}(1 \mathrm{e}+3 \mathrm{~h}) \cdot[23]$ Clear differences were observed, which could be understood from the combined effect of electron-hole exchange interaction and a splitting of the final state of $X^{2+}$, i.e. the p-shell triplet. Theoretical simulations with an atomistic model confirmed numerically the presence of such splitting. The physical origin of such splitting is that described in this work.

To illustrate this, in Fig. 5 we plot the emission spectrum from the ground state of $\mathrm{X}^{2-}$ and $\mathrm{X}^{2+}$ in a $\mathrm{QD}$ of $H=5 \mathrm{~nm}$. Electron-hole exchange is neglected again to focus on the effect of the hole p-shell splitting. For $\mathrm{X}^{2-}$, panel (a), the transition is schematically depicted in the inset. The final state has one electron in the s-shell and another in the p-shell. When considering spin degrees of freedom, the final state can be either singlet (S) or triplet (T). This gives rise to two optical resonances, split by the electron-electron exchange energy. The triplet resonance shows up at higher energies and is more intense, in agreement with Ref. [23] experiments. For $\mathrm{X}^{2+}$, panel (b), the triplet degeneracy is lifted (recall Fig. 2). Only $\mathrm{T}_{ \pm}$and $\mathrm{T}_{0}$ are optically accessible from the ground state, because the high-energy $p$ orbitals $\left(F_{z}= \pm 5 / 2\right)$ are unoccupied. As a result, the triplet resonance splits into two new resonances, a low-energy one associated with $\mathrm{T}_{0}$ and a high-energy one associated with $\mathrm{T}_{ \pm}$. The energy splitting, $\Delta_{1}$, is a fraction of $\mathrm{meV}$ and the $T_{ \pm}$resonance is more intense than the $T_{0}$ one. This is also in good agreement with Ref. [23] experiments (see Fig.2 therein).

\section{Conclusions}

We have shown that the p-shell of holes in circular QDs is split due to the valence band spin-orbit interaction coupling $\mathrm{p}_{+}$and $\mathrm{p}_{-}$HHs to LHs of different angular momenta. The larger the vertical aspect ratio, the larger 


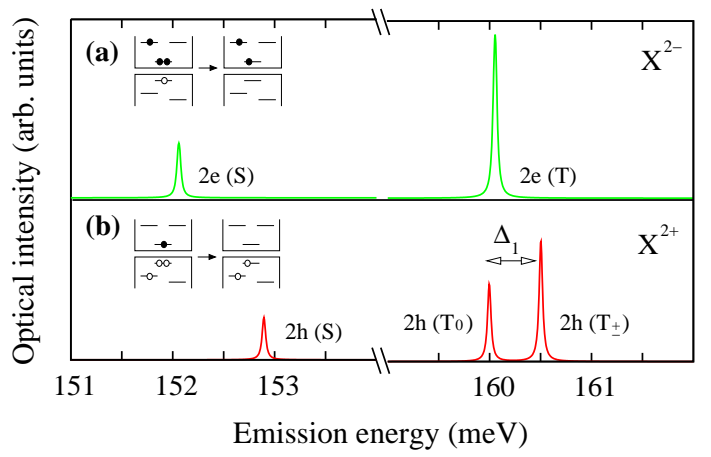

Figure 5: Emission from the ground state of excitons charged with two additional electrons (a) and two additional holes (b). The insets show the dominant electronic configurations of the initial and final states. The labels next to the resonances indicate the spin configuration. The energy excludes the bulk gap.

the splitting because the kinetic energy of LH decreases faster than that of $\mathrm{HH}$, so that the HH-LH coupling increases. This p-shell structure in turn gives rise to a splitting of the two-hole triplet sublevels, whose magnitude is also affected by electronic correlations. As a result, the optical spectrum of positively charged excitons (hot $\mathrm{X}^{+}, \mathrm{X}^{2+}$ ) reveals multiple resonances even in the absence of electron-hole exchange interaction. This is contrary to the expectation one would naively expect from usual single-band $\mathrm{HH}$ models, and agrees with experimental observations.

The spin of single holes and positive trions in InGaAs QDs with small aspect ratio is of high purity, and thus suitable for spin-based applications. In QDs with large aspect ratio, however, hot trions suffer from severe spin admixture due to strong HH-LH coupling.

Support from MICINN projects CTQ2008-03344 and CTQ2011-27324, UJI-Bancaixa project P1-1A2009-03 and the Ramon y Cajal program is acknowledged.

\section{References}

[1] S. Cortez, O. Krebs, S. Laurent, M. Senes, X. Marie, P. Voisin, R. Ferreira, G. Bastard, J. Gérard, T. Amand, Physical Review Letters 89 (2002) 207401. 
[2] S. Carter, Ş. Bădescu, A. Bracker, Physical Review B 81 (2010) 045305.

[3] M. Ware, E. Stinaff, D. Gammon, M. Doty, A. Bracker, D. Gershoni, V. Korenev, Ş. Bădescu, Y. Lyanda-Geller, T. Reinecke, Physical Review Letters 95 (2005) 177403.

[4] V. Jovanov, S. Kapfinger, M. Bichler, G. Abstreiter, J. Finley, Physical Review B 84 (2011) 235321.

[5] K. Kavokin, Physica Status Solidi (a) 195 (2003) 592-595.

[6] T. Warming, E. Siebert, A. Schliwa, E. Stock, R. Zimmermann, D. Bimberg, Physical Review B 79 (2009) 125316.

[7] M. Ware, A. Bracker, E. Stinaff, D. Gammon, D. Gershoni, V. Korenev, Physica E: Low-dimensional Systems and Nanostructures 26 (2005) 5558.

[8] E. Poem, J. Shemesh, I. Marderfeld, D. Galushko, N. Akopian, D. Gershoni, B. Gerardot, A. Badolato, P. Petroff, Physical Review B 76 (2007) 235304 .

[9] E. Siebert, T. Warming, A. Schliwa, E. Stock, M. Winkelnkemper, S. Rodt, D. Bimberg, Physical Review B 79 (2009) 205321.

[10] M. Scheibner, M. Yakes, A. Ponomarev, M. Doty, C. Hellberg, L. Whitman, T. Reinecke, D. Gammon, Nature Physics 4 (2008) 291-295.

[11] A. Schliwa, M. Winkelnkemper, D. Bimberg, Physical Review B 76 (2007) 205324.

[12] G. Bester, D. Reuter, L. He, A. Zunger, P. Kailuweit, A. Wieck, U. Zeitler, J. Maan, O. Wibbelhoff, A. Lorke, Physical Review B 76 (2007) 075338.

[13] M. Glazov, Journal of Physics: Condensed Matter 22 (2010) 025301.

[14] M. Doty, J. Climente, A. Greilich, M. Yakes, A. Bracker, D. Gammon, Physical Review B 81 (2010) 035308.

[15] D. Kim, S. Economou, Ş. Bădescu, M. Scheibner, A. Bracker, M. Bashkansky, T. Reinecke, D. Gammon, Physical Review Letters 101 (2008) 236804. 
[16] J. Planelles, W. Jaskólski, J. Aliaga, Physical Review B 65 (2001) 033306 .

[17] P. Sercel, K. Vahala, Physical Review B 42 (1990) 3690.

[18] J. Climente, J. Planelles, F. Rajadell, Journal of Physics: Condensed Matter 17 (2005) 1573.

[19] W. Press, S. Teukolsky, W. Vetterling, B. Flannery, Numerical Recipes in Fortran 77, Cambridge University Press, 1996.

[20] https://code.google.com/p/citool/, .

[21] I. Vurgaftman, J. Meyer, L. Ram-Mohan, Journal of Applied Physics 89 (2001) 5815 .

[22] F. Rajadell, J. Climente, J. Planelles, A. Bertoni, The Journal of Physical Chemistry C 113 (2009) 11268-11272.

[23] M. Ediger, G. Bester, B. Gerardot, A. Badolato, P. Petroff, K. Karrai, A. Zunger, R. Warburton, Physical Review Letters 98 (2007) 36808.

[24] R. Hanson, L. Kouwenhoven, J. Petta, S. Tarucha, L. Vandersypen, Reviews of Modern Physics 79 (2007) 1217.

[25] L. Woods, T. Reinecke, R. Kotlyar, Physical Review B 69 (2004) 125330.

[26] Y. Liao, J. Climente, S. Cheng, Physical Review B 83 (2011) 165317. 